\title{
Structure-activity Relationship of Pamamycins: Effect of Side Chain Length on Aerial Mycelium-inducing Activity
}

\author{
Ikuko Kozone $^{\dagger}$, Makoto Hashimoto ${ }^{\dagger \dagger}$, Udo Gräfe, Hiroshi Kawaide, Hiroshi Abe, \\ Masahiro Natsume
}

Received: December 20, 2007 / Accepted: February 16, 2008

(C) Japan Antibiotics Research Association

\begin{abstract}
Two pamamycin homologues with different side chain lengths were isolated from Streptomyces sp. HKI-0118. Aerial mycelium-inducing activity decreased by ca. $1 / 10$ per methylene unit in the side chain.
\end{abstract}

Keywords pamamycin, structure-activity relationship, aerial mycelium, differentiation, polyketide

Pamamycins are unique polyketides containing a nitrogen atom [1, 2]. Pamamycins were discovered as aerial mycelium-inducing substances of Streptomyces alboniger by McCann and Pogell [3], and their isolation and structural elucidation were accomplished by our group [4 6]. Pamamycin-607 (3), a major active component with MW 607, induces or stimulates aerial mycelium formation and regulates secondary metabolite production in many Streptomyces spp. in addition to the species in which it is produced [7].

We have examined the structure-activity relationship of pamamycins by isolating homologues $[8,9]$ and de- $N$ methyl derivatives from cultured material of $S$. alboniger $[10,11]$ and by preparing derivatives [12]. We have determined that the nitrogenous group is indispensable to aerial mycelium-inducing activity, that de- $N$-methylation increases this activity 1.5 times, and that substitution of the methyl group at $\mathrm{R}_{3}$ or $\mathrm{R}_{4}$ with an ethyl group markedly lowers activity.

To refine our understanding of the structure-activity relationship around the nitrogenous group, we planed to isolate homologues with different side chain lengths and to examine their aerial mycelium-inducing activity. There are two reports on such homologues: MS-282a and MS-282b were isolated from $S$. tauricus ATCC 27470 as inhibitors of calmodulin-activated myosin light chain kinase [13]. Härtl et al. reported the production of various lengths of the nitrogen-containing side chain of pamamycin $(n=2 \sim 4$ in Fig. 1) by Streptomyces sp. HKI-0118 and S. aurantiacus IMET 43917 [14], but they were unsuccessful in isolating these components.

We report the isolation of two pamamycin homologues, $\mathbf{1}$ and 2, with different side chain lengths from Streptomyces sp. HKI-0118 (Fig. 1) and describe their aerial myceliuminducing activity.

Streptomyces sp. HKI-0118 was cultured in 2.0-liter Erlenmeyer flasks containing 1.0 liter of glucose-oat meal-yeast extract medium [14] at $28^{\circ} \mathrm{C}$ for 7 days on a rotary shaker (150 rpm). The fermentation broth (26 liters) was separated into filtrate and mycelia, then the filtrate was treated with EtOAc at $\mathrm{pH} 8$ and the mycelia were macerated in $\mathrm{Me}_{2} \mathrm{CO}$. The $\mathrm{Me}_{2} \mathrm{CO}$ extract of mycelia was
M. Natsume (Corresponding author), H. Kawaide, H. Abe: Institute of Symbiotic Science and Technology, Tokyo University of Agriculture and Technology, Saiwai-cho, Fuchu, 183-8509 Tokyo, Japan, E-mail: natsume@cc.tuat.ac.jp

I. Kozone, M. Hashimoto: United Graduate School of Agricultural Science, Tokyo University of Agriculture and Technology, Saiwai-cho, Fuchu, 183-8509 Tokyo, Japan
U. Gräfe: Hans-Knöll-Institute of Natural Products Research, Jena, Germany

${ }^{\dagger}$ Present address: Chemical Biology Team, Biological Information Research Center (BIRC), National Institute of Advanced Industrial Science and Technology (AIST), Aomi, Koto-ku, Tokyo 135-0064, Japan

${ }^{\dagger}$ Present address: Biotechnology Research Center, Toyama Prefectural University, Imizu, Toyama 939-0398, Japan 
concentrated in vacuo and the aqueous residue was washed with $n$-hexane and then treated with EtOAc at $\mathrm{pH}$ 8. EtOAc extracts obtained from the culture filtrate and mycelia were dissolved in small amounts of EtOAc, combined and then applied to a silica gel column. The column was washed with EtOAc, then the solvent in the column was substituted with EtOAc- $n$-hexane $(2: 8)$, and the pamamycins were eluted with EtOAc - $n$-hexane - diisopropylamine $(2: 8: 0.5)$ to yield $257 \mathrm{mg}$ of crude pamamycin mixture. This mixture was then purified by preparative ODS-HPLC (Fig. 2(a)). Pamamycins are separated by ODS-HPLC according to

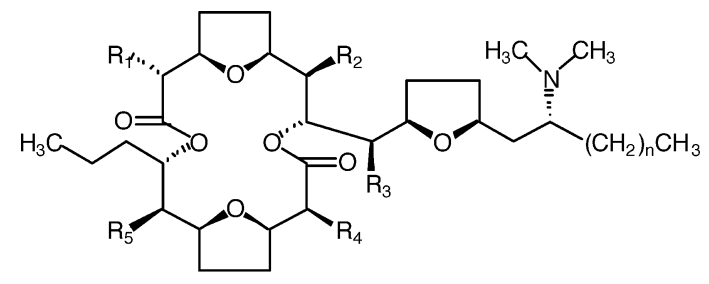

$$
\begin{aligned}
& \text { Bishomopamamycin-635A }(1: \mathrm{n}=4) \\
& \text { Homopamamycin-621A }(2: \mathrm{n}=3) \\
& \text { Pamamycin-607 }(3: \mathrm{n}=2)
\end{aligned}
$$

Fig. 1 Structures of pamamycin-607 (3) and its side chain homologues.

$\mathrm{R}_{1 \sim 4}=\mathrm{CH}_{3}$ and $\mathrm{R}_{5}=\mathrm{H}$ in all compounds. their molecular weight [9]. EI-MS of the peak eluting at 17.8 minutes showed an $[\mathrm{M}]^{+}$ion at $m / z \quad 635$ and two clusters of $\alpha$-cleavage ions of the dimethylamino group at $\mathrm{m} / z$ 100, 114 and 128 and at $\mathrm{m} / z$ 592, 578 and 564 (Fig. 3). These results show that the peak was composed of three side chain homologues with MW 635. These homologues were separated by HPLC with two $\mathrm{NH}_{2}$-columns connected in series (Fig. 2(b)). Collection and mass spectral analysis of each peak showed that the major peak at Rt 16.8 minutes showed the $m / z 128$ ion, which was expected to be the side chain homologue with 2 additional methylene units compared to pamamycin-607. Repeated collection of the peak afforded compound $\mathbf{1}$ (yield $4.33 \mathrm{mg}$ ). The side chain homologue of pamamycin-607 with MW 621 (2) was isolated from the peak at Rt 16.0 minutes during ODSHPLC (Fig. 2(a)) and by subsequent $\mathrm{NH}_{2}$-HPLC (Rt 19.8 minutes in Fig. 2(c)) (yield $1.16 \mathrm{mg}$ ).

Alkyl substituents at $R_{1} \sim R_{5}$ of the isolated homologues were elucidated by capillary GC-MS analysis of the two diol products obtained by $\mathrm{LiAlH}_{4}$ degradation of each homologue by the method reported previously [8 10]. Both homologues had the same substituents, $\mathrm{R}_{1} \sim \mathrm{R}_{5}$, as pamamycin-607, and were named bishomopamamycin635A (1, MW 635) and homopamamycin-621A (2, MW 621). The planar structure of compound $\mathbf{1}$ is the same as that of MS-282a [13], however the identity of the two

(a)

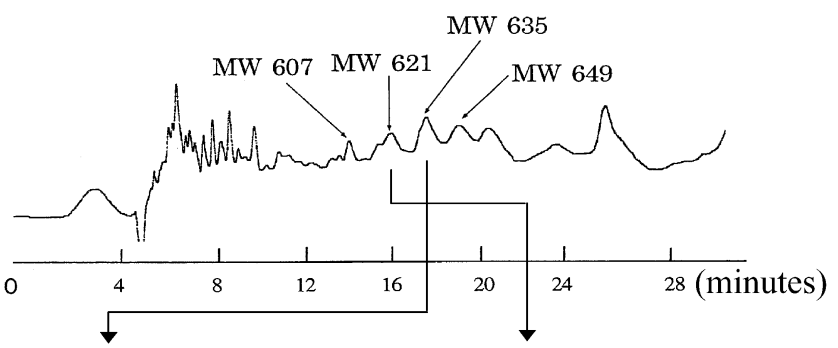

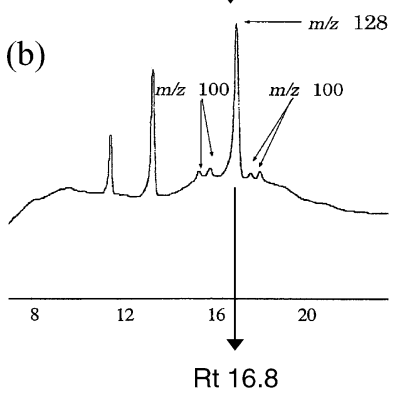

(1)

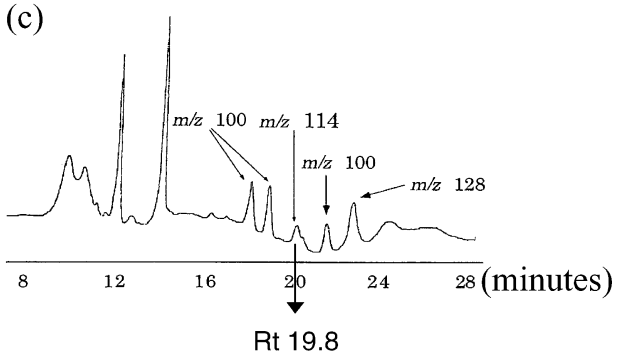

(2)

Fig. 2 Purification of side chain homologues of pamamycin by HPLC.

Chromatographic conditions: (a); Column, Develosil ODS-5 (250×20 mm); solvent, $0.2 \%$ aq. $\mathrm{NH}_{4} \mathrm{OAc}-\mathrm{MeOH}(1: 9)$; flow rate, $10 \mathrm{ml} /$ minute; detection, UV at $225 \mathrm{~nm}$, (b) and (c); column, Develosil $\mathrm{NH}_{2}-5(250 \times 8 \mathrm{~mm}) \times 2$ (connected in series); solvent, MeOH $n$-hexane- $n$ - $\mathrm{BuNH}_{2}$ (5:100:5); flow rate, $2.0 \mathrm{ml} /$ minute; detection, UV at $235 \mathrm{~nm}$. Numerals in (a) show the molecular weights of the peak components and those in (b) and (c) show the $\mathrm{m} / \mathrm{z}$ values of the smaller fragment ion cleaved at the a position of nitrogen (ion a in Fig. 3), these were determined by El-MS. 


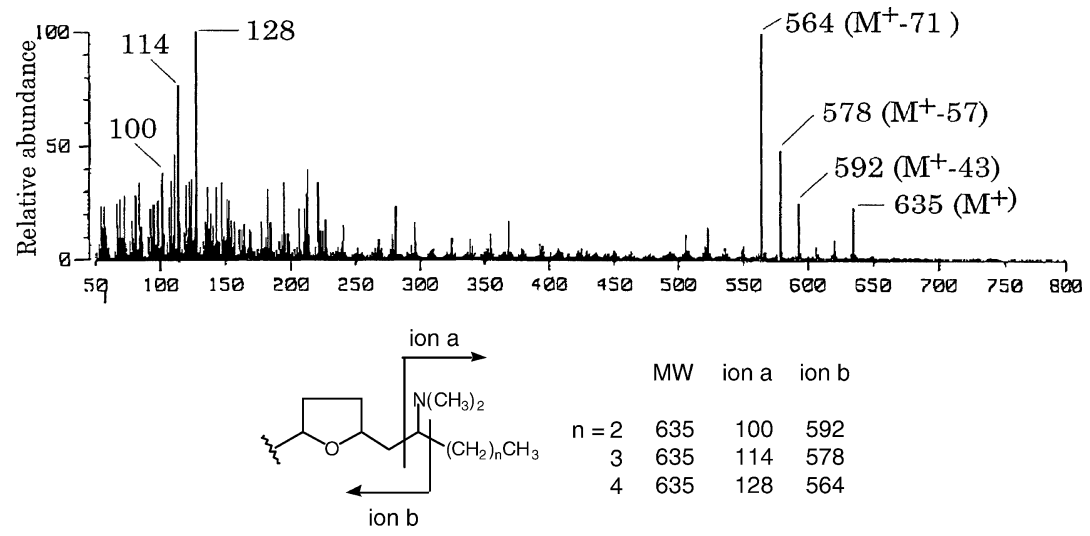

Fig. 3 El-MS spectrum of the peak with Rt 17.8 minutes in ODS-HPLC.

Table 1 Aerial mycelium-inducing activity of side chain homologues of pamamycin

\begin{tabular}{|c|c|c|c|}
\hline \multirow{3}{*}{ Compound } & \multicolumn{3}{|c|}{$\begin{array}{l}\text { Diameter of aerial mycelium-inducing zone }(\mathrm{mm}) \\
\text { (Diameter of growth inhibitory zone }(\mathrm{mm}))\end{array}$} \\
\hline & \multicolumn{3}{|c|}{ Dose ( $\mu \mathrm{g} /$ disc) } \\
\hline & 1 & 3 & 10 \\
\hline Bishomopamamycin-635A (1) & - $^{*}(-)$ & $-(-)$ & - (18) \\
\hline Homopamamycin-621A (2) & $-(-)$ & $14(-)$ & $34(19)$ \\
\hline Pamamycin-607 (3) & $40(-)$ & $42(21)$ & $55(34)$ \\
\hline
\end{tabular}

* Aerial mycelium-inducing or growth-inhibitory zone was not observed around paper disc (8 mm diameter).

compounds could not be confirmed as described later. The relative stereochemistries of $\mathbf{1}$ and $\mathbf{2}$ are thought to be the same as that of pamamycin-607, because the smaller diol fragment obtained from $\mathbf{1}$ or $\mathbf{2}$ by $\mathrm{LiAlH}_{4}$ degradation showed an identical retention time and fragmentation pattern to that of pamamycin-607 by capillary GC-MS analysis.

The side chain of pamamycin-607 is derived from acetate units [2]. There has, however, been no discussion as to whether the side chains of MS-282a and b [13] or pamamycin homologues [14] are linear or branched alkyl groups. The side chain of 2 was proven to be $n-\mathrm{C}_{4} \mathrm{H}_{9}$, because a strong isotope peak was observed at $\mathrm{m} / \mathrm{z} 115$ following EI-MS analysis of the crude pamamycin fraction obtained from $\left[1-{ }^{13} \mathrm{C}\right]$-propionate-treated cultured material. This result shows that the side chain of homopamamycin $\mathbf{2}$ was developed from propionate as the starter unit of the polyketide chain. Although the number of acetate units incorporated into $\mathbf{1}$ was uncertain based on feeding experiments with ${ }^{13} \mathrm{C}$-acetate, the side chain of $\mathbf{1}$ should be linear because the ratio of homologues with $n=2,3$ and 4 produced was similar in the crude pamamycin fraction obtained from inorganic salt-starch agar (ISS) medium and glucose - oat meal - yeast extract medium. Glucose - oat meal-yeast extract medium is thought to be rich in branched short chain fatty acids or their precursors, branched chain amino acids, while ISS medium is not.

Aerial mycelium-inducing activities of $\mathbf{1}$ and $\mathbf{2}$ were examined using a paper disc method with $S$. alboniger NBRC 12738 on Hickey and Tresner's agar medium containing cerulenin [11]. Activity decreased to $c a$. 1/10 for each methylene unit in the side chain (Table 1).

When these results are taken together with previous knowledge [8 12], the structure-activity relationship of pamamycins can be summarized as shown in Fig. 4. With an increase in bulkiness on the right side of the molecule, aerial mycelium-inducing activity markedly decreases: e.g. a change from $\mathrm{CH}_{3}$ to $\mathrm{C}_{2} \mathrm{H}_{5}$ at alkyl substituents $\mathrm{R}_{3}$ or $\mathrm{R}_{4}$ caused loss of activity [9] and an increase in the number of methylene units (n) decreased activity by $c a$. 1/10 per methylene unit. The L shaped conformation is important for the activity because opening of the macrodiolide ring or scission into two constituent hydroxy acids resulted in a marked drop in activity $[5,8,9,15]$. These results indicate 


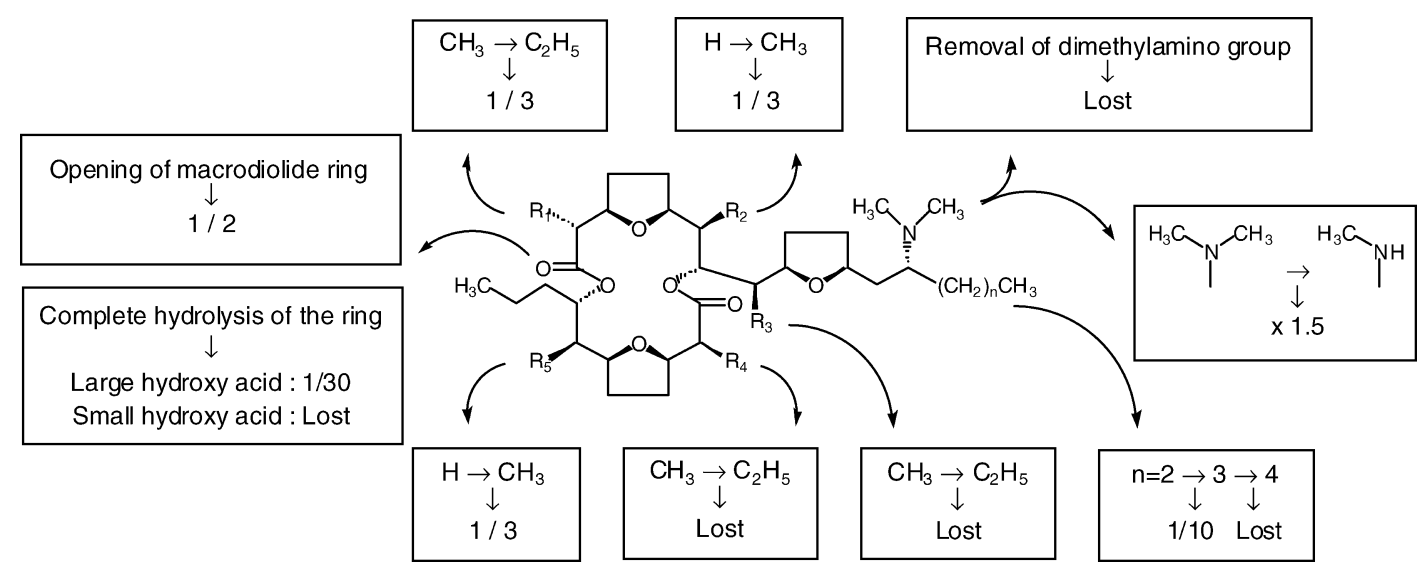

Fig. 4 Relationship between structure and aerial mycelium-inducing activity in pamamycins.

Upper lines in boxes summarize structural change and bottom lines the change in activity due to the structural change. "1/10" indicates that the product showed $1 / 10$ th the activity of the original compound, " $\times 1.5$ " that the product showed 1.5 times stronger activity than the original compound, and "lost" that the product lost all activity.

the presence of a receptor molecule and that the binding of pamamycin to this receptor triggers the onset of aerial mycelium formation.

Pamamycins show growth inhibitory activity against $S$. alboniger at high doses. Side chain homologues $\mathbf{1}$ and $\mathbf{2}$ showed comparable activity, which was weaker than that of pamamycin-607 (3) (Table 1). Similar results were observed in homologues with different $\mathrm{R}_{1} \sim \mathrm{R}_{5}$ [12]; a specific relationship is not present between the partial structure and growth inhibitory activity. Gräfe et al. showed that pamamycin acts as a phase-transfer mediator conveying anions or as a protonophore [15 18]. These characteristics were attributed to the lipophilicity of the molecule and did not involve a particular partial structure. Thus, growth inhibitory activity of pamamycin must be derived from its protonophoric or anion-transferring ability.

We tried to re-isolate MS-282a and b from $S$. tauricus JCM 4837 (=ATCC 27470), but could not detect their production either in the medium that was used for the production of pamamycins in S. alboniger [9] or in ISS medium, in which abundant aerial mycelia formed [7] (data not shown). Nakanishi et al. showed that the production of MS-282a and b was greatly affected by the nitrogen source and that the amount of soluble vegetable protein was the most important factor [13]. Considering this information and the finding that the side chain homologues with $n=3$ or $\mathrm{n}=4$ showed weak or no activity, MS-282a and $\mathrm{b}$ may not be involved in aerial mycelium formation in S. tauricus. On the other hand, Streptomyces sp. HKI-0118 produced aerial mycelia on oat meal agar medium or ISS agar medium [7] and production of pamamycin with $n=2$ was confirmed in oat meal medium in this experiment and in ISS medium in the former experiment [7]. These results show that endogenously produced pamamycins play a role in aerial mycelium formation in Streptomyces sp. HKI-0118.

Acknowledgments Streptomyces sp. HKI-0118 was kindly provided by the late Dr. Gräfe of Hans-Knöll-Institute of Natural Products Research, Germany. This work was initiated in response to his request for technical assistance on the separation of pamamycin homologues and was developed in a coordinated study. We would like to express our sincere thanks to Dr. Gräfe, who cowrote this paper, despite the fact that his agreement could not be obtained for its final form and that the isolated pamamycin homologues could not be tested using his bioassay system.

\section{References}

1. Hashimoto M, Komatsu H, Kozone I, Kawaide H, Abe H, Natsume M. Biosynthetic origin of the carbon skeleton and nitrogen atom of pamamycin-607, a nitrogen-containing polyketide. Biosci Biotech Biochem 69: 315-320 (2005)

2. Hashimoto M, Kozone I, Kawaide H, Abe H, Natsume M. Biosynthetic route of pamamycin-607, a nitrogen-containing polyketide. J Antibiot 58: 722-730 (2005)

3. McCann PA, Pogell BM. Pamamycin: a new antibiotic and stimulator of aerial mycelia formation. J Antibiot 32: 673-678 (1979)

4. Kondo S, Yasui K, Natsume M, Katayama M, Marumo S. Isolation, physico-chemical properties and biological activity of pamamycin-607, an aerial mycelium-inducing substance from Streptomyces alboniger. J Antibiot 41: 1196-1204 (1988)

5. Kondo S, Yasui K, Katayama M, Marumo S, Kondo T, 
Hattori H. Structure of pamamycin-607, an aerial myceliuminducing substance of Streptomyces alboniger. Tetrahedron Lett 28: 5861-5864 (1987)

6. Natsume M, Kondo S, Marumo S. The absolute stereochemistry of pamamycin-607, an aerial myceliuminducing substance of Streptomyces alboniger. J Chem Soc Chem Commun 1989: 1911-1913 (1989)

7. Hashimoto M, Kondo T, Kozone I, Kawaide H, Abe H, Natsume M. Relationship between response to and production of the aerial mycelium-inducing substances pamamycin-607 and A-factor. Biosci Biotech Biochem 67: 803-808 (2003)

8. Natsume M, Yasui K, Kondo S, Marumo S. The structures of four new pamamycin homologues isolated from Streptomyces alboniger. Tetrahedron Lett 32: 3087-3090 (1991)

9. Natsume M, Tazawa J, Yagi K, Abe H, Kondo S, Marumo S. Structure-activity relationship of pamamycins: effects of alkyl substituents. J Antibiot 48: 1159-1164 (1995)

10. Kozone I, Chikamoto N, Abe H, Natsume M. De- $N-$ methylpamamycin-593A and B, new pamamycin derivatives isolated from Streptomyces alboniger. J Antibiot 52: 329-331 (1999)

11. Kozone I, Abe H, Natsume M. Effects of $N$-demethylation of pamamycins on aerial mycelium-inducing and growth inhibition activities. J Pesticide Sci 26: 149-153 (2001)

12. Natsume M, Honda A, Oshima Y, Abe H, Kondo S, Tanaka F, Marumo S. Roles of the dimethylamino group and macrodiolide ring of pamamycin-607 in its aerial myceliuminducing activity. Biosci Biotech Biochem 59: 1766-1768 (1995)

13. Nakanishi S, Kita K, Uosaki Y, Yoshida M, Saitoh Y, Mihara A, Kawamoto I, Matsuda Y. MS-282a and MS-282b, new inhibitors of calmodulin-activated myosin light chain kinase from Streptomyces tauricus ATCC 27470. J Antibiot 47: 855-861 (1994)

14. Härtl A, Stelzner A, Schlegel R, Heinze S, Hülsmann H, Fleck W, Gräfe U. Discovery of new homologous pamamycins by mass spectrometry and post mortem inhibitory action on autolysis of chicken embryo chorioallantoic membrane blood vessels. J Antibiot 51: 1040-1046 (1998)

15. Natsume M. Differentiation of aerial mycelia - Pamamycins and calcium ion in Streptomyces alboniger-. Actinomycetologica 13: 11-19 (1999)

16. Stengel C, Reinhardt G, Gräfe U. A simple screening procedure for microbial phase-transfer mediators conveying anions. J Basic Microbiol 32: 339-345 (1992)

17. Gräfe U, Stengel C, Möllmann U, Heinisch L. Formation of lipopilic ion pairs by derivatives of pamamycin. Pharmazie 49: 343-346 (1994)

18. Grigoriev P, Berg A, Schlegel R, Gräfe U. Protonophoric activities of helioferin and pamamycin, lipophilc tertiary amine antibiotics from Mycogone rosea and Streptomyces aurantiacus. Bioelectrochem Bioenerget 39: 295-298 (1996) 